\title{
Analysis of the Reduction of Stability in Partially Filled Tank Vehicles
}

\author{
Gonzalo G. MORENO CONTRERAS*, Juan Carlos SERRANO, Bladimir RAMÓN VALENCIA
}

\begin{abstract}
The accidents of heavy vehicles due to stability problems are not so frequent, but when this type of accident occurs, its results are often fatal, which is why the analysis of stability of heavy vehicles allows us to predict their limit of speed to safely travel on road or perform certain maneuvers that involve rollover risk. Additionally, when these vehicles transport liquids, the stability problems may be greater. Taking into account that one of the main factors to determine the risk of accident due to rollover is the Static Rollover Threshold, this article develops this factor for road tankers and it is determined by a case study such as these loads depending on the filling of the tank; they affect the stability of this type of vehicles and a comparison is made with respect to the improvement of the stability if the movement of this load is avoided.
\end{abstract}

Keywords: road safety; road tanker; stability; static rollover threshold

\section{INTRODUCTION}

The movement of the load in road tankers is the reason why the driver must take special care when performing certain maneuvers, since this movement significantly alters the load distribution of the vehicle and its performance [1]. When these vehicles are performing a curved trajectory, the inertial forces present due to the vehicle's loads make it prone to rollover, which will be more critical in the presence of factors such as liquid loads that can affect this type of accident related to stability vehicle.

According to this, the Static Rollover Threshold (SRT) is one of the main factors that allows to determine how stable a vehicle is when it makes a curve, which by means of a quasi-static balance of the forces acting on the vehicle and the limit of these determines the vehicle's stability factor $[2,3]$. Additionally, this factor also allows predicting the maximum speed with which the vehicle can take a curve of a certain radius without risk of rollover [4]. Taking all these aspects into account, it is important for several researchers to determine how the tank filling percentage and the movement of the transported fluid can affect the calculation of the stability factor [5-7]. Therefore, the present article makes a two-dimensional analysis of the loads movement in a tank of circular cross section under the action of a lateral acceleration (Section 2). In Section 3 , taking into account the model developed by [8], the calculation of the stability factor for this type of vehicles is made. In Section 4, a case study analyzes how the filling level of the tank can affect the stability factor. In addition a comparison is made with respect to the possibility of implementing a mechanism that makes the lateral movement of the load impossible, and finally, the conclusions of the work carried out are detailed.

\section{MOVEMENT OF FLUID WITH UNIFORM ACCELERATION}

The equations that describe the linear movement of an incompressible fluid inside a circular tank subjected to uniform acceleration (Fig. 1) are the continuity equations and the momentum conservation equation [9], which is presented in Eq. (1) and Eq. (2).

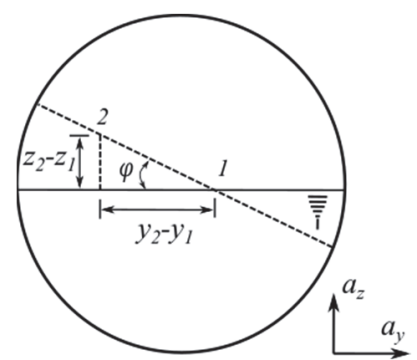

Figure 1 Fluid in acceleration in tank of circular cross section

$\nabla \cdot v=0$

and

$\rho\left(\frac{\partial v}{\partial t}+v \cdot \nabla v\right)=-\nabla P+\rho g+\mu \nabla^{2} v$

where $v$ is the fluid velocity $(\mathrm{m} / \mathrm{s}), P$ is the fluid pressure $(\mathrm{Pa}), \rho$ is the fluid density $\left(\mathrm{kg} / \mathrm{m}^{3}\right), \mu$ is the viscosity of the fluid (Pa.s), and $g$ is the acceleration due to gravity $\left(\mathrm{m} / \mathrm{s}^{2}\right)$. When the fluid is subjected to a lateral acceleration, each element of the fluid in the tank experiences the same acceleration, besides its velocity $v$ is a function of time but not of the position, Eq. (2) can be rewritten as:

$\rho \frac{D v}{D t}=-\nabla P+\rho g+\mu \nabla^{2} v$

where $D / D t$ is the substantial derivative. For a uniformly accelerated flow $D v / D t=a$ and $\nabla^{2} v=0$, which reduces Eq. (3) to:

$\rho a=-\nabla P+\rho g$

Eq. (4) can be expressed explicitly as:

$\frac{\partial P}{\partial x} \vec{i}+\frac{\partial P}{\partial y} \vec{j}+\frac{\partial P}{\partial z} \vec{k}+\rho g \vec{k}=-\rho\left(a_{x} \vec{i}+a_{y} \vec{j}+a_{z} \vec{k}\right)$

Considering the movement of the fluid in the $y-z$ plane, Eq. (5) is reduced to: 
$\mathrm{d} P=-\rho a_{y} \mathrm{~d} y-\rho\left(g+a_{z}\right) \mathrm{d} z$

When a tank is uniformly accelerated in the $y$ direction, initially the free surface will move back and forth. After some time this movement disappears, passing from this transient state to a uniformly accelerated movement. The position of the liquid surface in a uniformly accelerated tank is shown in Fig. 1. For $\rho=c t e$, the pressure difference between two points 1 and 2 in the fluid is determined by integration, so that the Eq. (6) becomes:

$$
P_{2}-P_{1}=-\rho a_{y}\left(y_{2}-y_{1}\right)-\rho\left(g+a_{z}\right)\left(z_{2}-z_{1}\right)
$$

The vertical increase of the free surface at point 2 relative to point 1 can be determined by selecting these points on the free surface where $P_{2}=P_{1}$ and solving Eq. (7).

$$
\Delta z=z_{2}-z_{1}=-\frac{a_{y}}{g+a_{z}}\left(y_{2}-y_{1}\right)
$$

In an in-compressible fluid with uniform acceleration in linear motion, the lines of constant pressure are parallel to the surface whose slope in the $y-z$ plane with $a_{z}=0$ can be determined by:

slope $=-\frac{a_{y}}{g+a_{z}}=-\frac{a_{y}}{g}=\tan \varphi$

In order to determine the fluid center of gravity $\left(C G_{2}\right)$ of a tank of circular cross-section, subject to uniform acceleration and with a certain percentage of filling, the coordinates of the center of gravity are determined by the solution of:

$\bar{x}=\frac{1}{A} \iint_{A} x \mathrm{~d} A$

$\bar{y}=\frac{1}{A} \iint_{A} y \mathrm{~d} A$

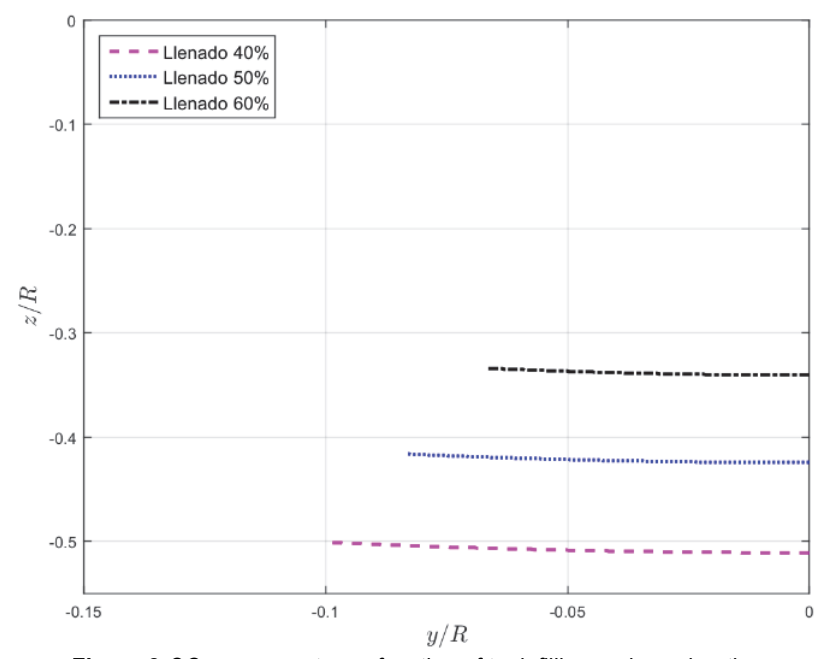

Figure $2 \mathrm{CG}_{2}$ movement as a function of tank filling and acceleration
Fig. 2 shows the variation of $\left(\mathrm{CG}_{2}\right)$ for different filling percentages $(40 \%, 50 \%$ and $60 \%)$ as a function of the acceleration $0 \leq a_{y} \leq 0.3 \mathrm{~g}$. It is observed that for greater accelerations, the $\left(C_{2}\right)$ moves backwards and upwards in pendulum form [10]. It is also observed that with lower filling percentages the $\left(C G_{2}\right)$ has a greater oscillation, which influences the stability of vehicle.

\section{VEHICLE STABILITY ANALYSIS}

For this analysis, the two-dimensional representation of the road tanker with circular cross section was used (Fig. 3 - Section AA), in which it can be seen that the vehicle has a fixed center of gravity $\left(C G_{1}\right)$, which corresponds to the non-mobile weight of the vehicle $\left(w_{1}\right)$ (in the figures, the $C G_{1}$ was located above the geometric center of the tank, in order to better observe its movement, but in reality, it is below), and a moving center of gravity $\left(C G_{2}\right)$, which corresponds to the transported fluid weight $\left(w_{2}\right)$. It is assumed by what is specified in section 2 that the movement of the fluid is linear. In order to be able to observe the movement of the fixed weight of the vehicle $\left(C G_{1}\right)$, a large height was assumed in the figures.

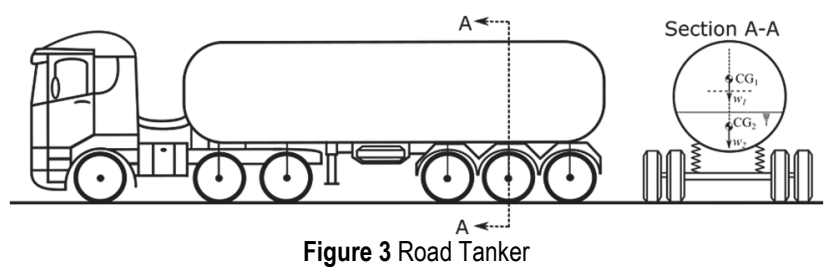

Initially, when the vehicle makes a curved trajectory (in this case to the right) it can be observed that the centripetal inertial forces $\left(m_{1} a_{y}\right.$ and $\left.m_{2} a_{y}\right)$ appear in the respective centers of gravity (Fig. 4). These forces make that the vehicle, by action of the stiffness of the tires and the suspension, inclines at an angle $\theta$, which causes the movement of the $C G_{1}$ around the center of rotation of the vehicle $(C R)$; then, the transported liquid assumes its new horizontal position, which makes that the $C G_{2}$ moves with respect to its initial position, making a movement similar to that of a pendulum around the geometric center of the tank.

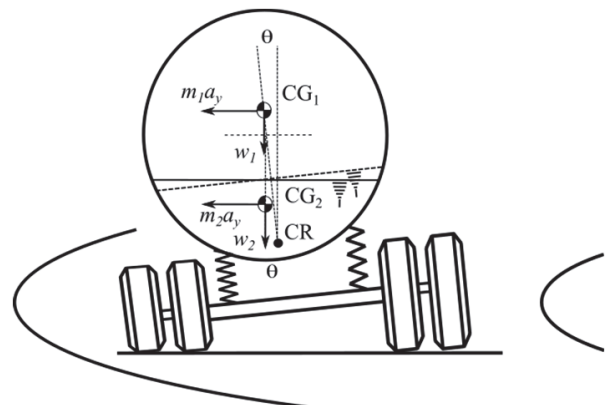

Figure 4. Road tanker, under the action of the angle of inclination $(\theta)$

Additionally, due to the linear movement of the fluid as a result of its lateral acceleration, the center of gravity of the mobile load $\left(C G_{2}\right)$ moves at a greater angle $\left(\theta_{1}\right)$, first due to the inclination of the vehicle (Fig. 4) and second due to the movement of the transported fluid. 


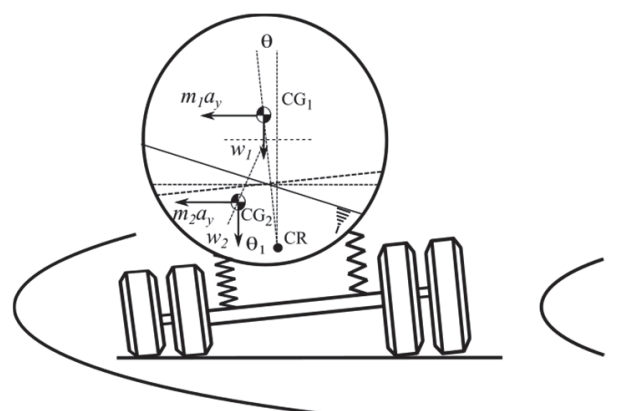

Figure 5 Road tanker, under the action of the transported fluids movement

Taking into account these specifications of movement of the centers of gravity, and making a development similar to that described in [8], the two-dimensional model of the vehicle (Fig. 6a) is represented by a kinematic model (Fig. $6 \mathrm{~b})$. Once the model is made and using the Davies Method as an analysis tool [11-13], it is possible to obtain the vehicle stability factor $(S R T)$ and the forces acting in each of the systems of the developed model (suspension and tires).

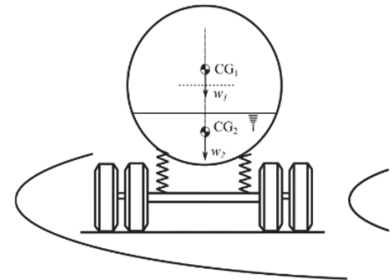

(a)

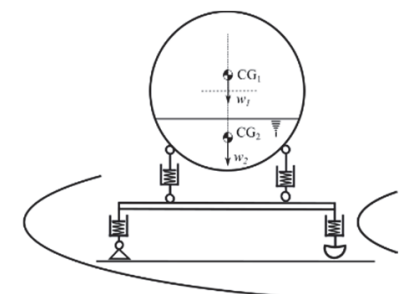

(b)
Figure 6 (a) Two-dimensional model of the vehicle. (b) Kinematic model of the vehicle

From the quasi-static analysis of the model, which uses the point of contact of the wheel outside the curve with the ground (point A) (Fig. 7), the following moment equation is obtained.

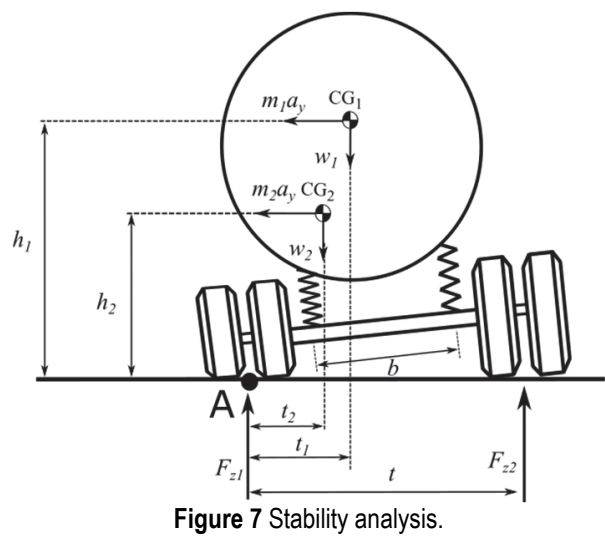

From Eq. 12 and Fig. 7 we have that: $\mathrm{t} 1$ is the lateral distance from the contact point of the external wheel to the curve up to $C G_{1}, t_{2}$ is the lateral distance from the point of support of the external wheel to the curve to the $C G_{2}, h_{1}$ is the height of the $C G_{1}, h_{2}$ is the height of the $C G_{2}, \mathrm{t}$ is the lateral distance between the wheels or track width, $b$ is the lateral distance of separation of the suspension, and $F_{z i}$ are the normal forces of vehicle support. Taking into account that in the threshold of rollover of the vehicle, the normal force of the internal wheel to the curve reaches zero $\left(F_{z 2}=\right.$ $0)$, then: $m_{1} g t_{1}+m_{2} g t_{2}-m_{1} a_{y} h_{1}-m_{2} a_{y} h_{2}=0$

By rearranging Eq. 13, the two-dimensional stability factor $(S R T)$ for a road tanker is expressed by the following equation:

$$
S R T=\frac{a_{y}}{g}=\frac{m_{1} t_{1}+m_{2} t_{2}}{m_{1} h_{1}+m_{2} h_{2}}
$$

This equation tells us that the stability factor does not only depend on the position of the centers of gravity, but also on the fixed weight of the vehicle and the weight of the liquid transported.

\section{CASE STUDY}

For this study we used the model of a road tanker (Fig. 3 ) with the following specifications:

Table 1 Vehicle characteristics

\begin{tabular}{|l|l|l|}
\hline \multicolumn{3}{|c|}{ Road tanker } \\
\hline Parameters & Value & Units \\
\hline Weight of empty vehicle $\left(w_{1}\right)$ & 60 & $\mathrm{kN}$ \\
\hline Weight of transported liquid $\left(w_{2}\right)$ - (water) & 407.66 & $\mathrm{kN}$ \\
\hline Combined stiffness of suspension - (3 axles) & 5400 & $\mathrm{kN} / \mathrm{m}$ \\
\hline Combined stiffness of tires & 5040 & $\mathrm{kN} / \mathrm{m}$ \\
\hline $\begin{array}{l}\text { Side distance between the wheels or track width } \\
(t)\end{array}$ & 1.86 & $\mathrm{~m}$ \\
\hline Tank diameter & 2.03 & $\mathrm{~m}$ \\
\hline$C G_{1}$ initial height $\left(h_{1}\right)$ & 0.85 & $\mathrm{~m}$ \\
\hline Initial height of the tanks geometric center & 2.125 & $\mathrm{~m}$ \\
\hline Lateral separation distance of the suspension $(b)$ & 0.95 & $\mathrm{~m}$ \\
\hline
\end{tabular}

For the calculation of the stability factor, ISO-14792 Steady State Circular Test [14] was taken into account and for road safety the lateral load transfer must not be greater than $60 \%[15,16]$ (For this reason, the results of the $S R T$ factor are lower than those reported in other research. Once the model was implemented, the centripetal forces $\left(m_{1} a_{y}\right.$ and $m_{2} a_{y}$ ) were gradually increased until the lateral load transfer was the recommended one. In Fig. 8 you can see how initially the stability of the vehicle is quite high, but as the tank is filled and the weight of the liquid $\left(w_{2}\right)$ increases, the stability factor decreases markedly, since the height of its center of gravity $\left(h_{2}\right)$ is gradually increasing. Additionally, it can be observed that the angle of inclination of the vehicle due to the action of the stiffness of the suspension and the tires (Body roll angle - $\theta$ ) increases with the filling of the tank.

In Fig. 9 it can be seen how the movement of the fluid decreases the stability factor of the vehicle with respect to the same vehicle but which is not allowed to move the fluid.

$w_{1} t_{1}+w_{2} t_{2}-m_{1} a_{y} h_{1}-m_{2} a_{y} h_{2}-F_{z 2} t=0$

As can be seen from Fig. 9 and Fig. 10 the stability is mostly affected for tank filling levels between $40 \%$ and $60 \%$, meaning an average reduction of the stability factor of $0: 01356$. 


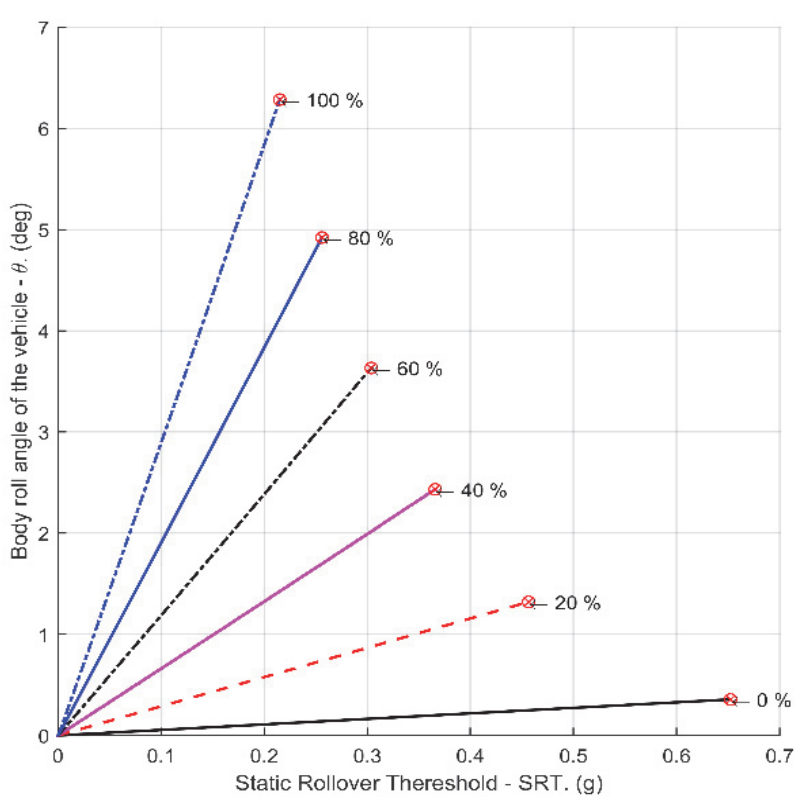

Figure 8 Stability factor
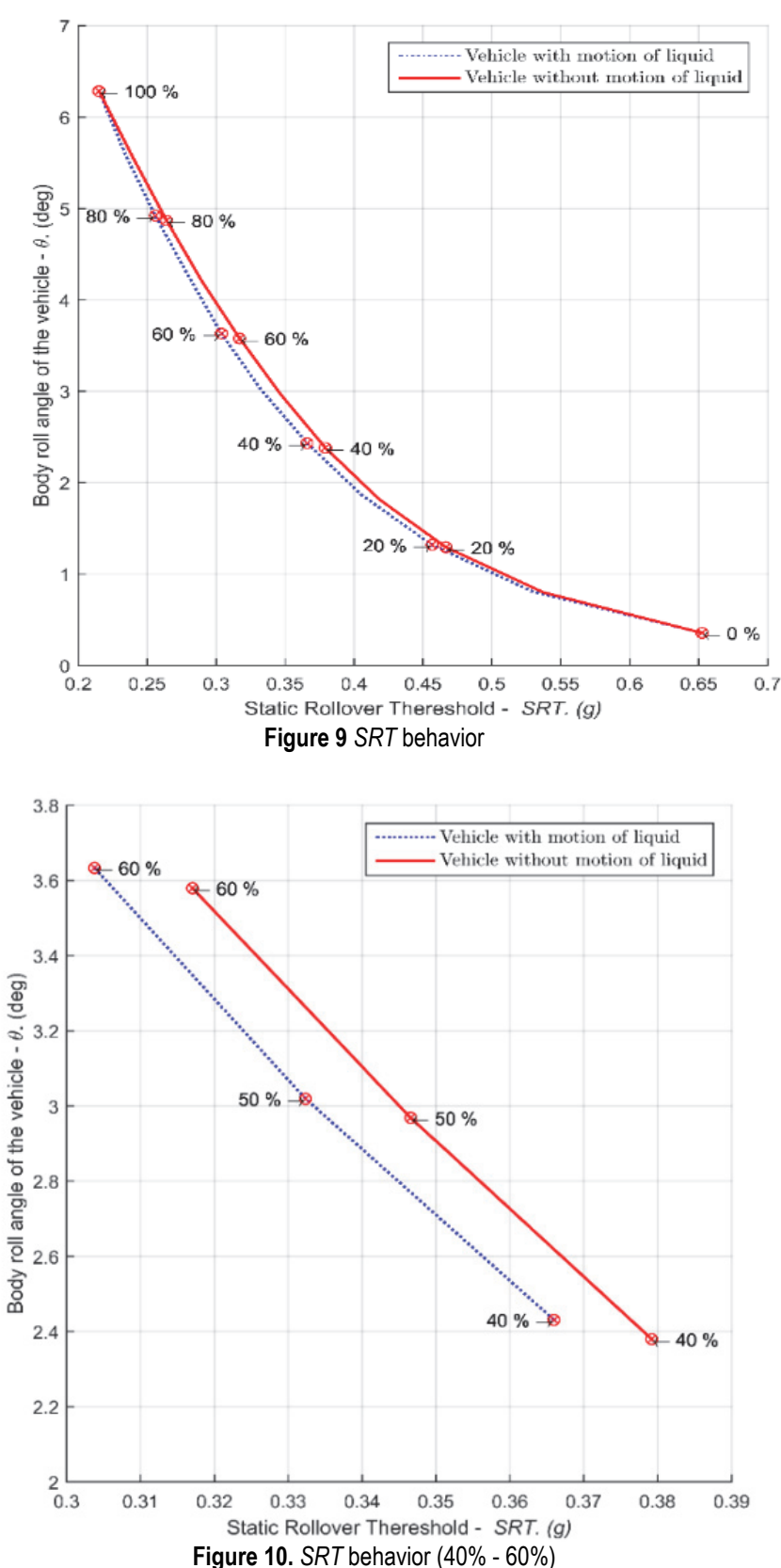

From Eq. (14), and as indicated in Eq. (15), it is known that the stability factor can be expressed as a function of the velocity of the vehicle $(V)$ when taking a curve and the radius of the curve $(R)$. Therefore, for a vehicle with fluid movement and without fluid movement, and with a tank filling level of $50 \%$, its stability factor passes $S R T=0: 3466$ to $S R T=0: 3323$ respectively; these values of $S R T$ factor in a curve of $R=100 \mathrm{~m}$ represent a speed reduction of approximately $2.4 \mathrm{~km} / \mathrm{h}$. This is really important to detail, since this allows to see the need to develop mechanisms that prevent the lateral movement of the fluid, type breakwater, which would increase the stability of the vehicle and therefore the road safety.

$$
S R T=\frac{a_{y}}{g}=\frac{V^{2}}{R g}
$$

\section{CONCLUSIONS}

In the present work, the stability factor for car tank type vehicles was determined. It can be evidenced that this factor is not only a function of the location of the center of gravity of the vehicle, but it is also a function of the fixed and mobile weight. vehicle.

In the results of the test performed, it was possible to show that as the vehicle acquires a greater load, the stability decreases. This is due to the fact that the height $\left(h_{2}\right)$ of the center of gravity of the liquid weight of the vehicle increases.

Additionally, it was determined that the stability of the vehicles can be increased, if mechanisms are developed that prevent the lateral movement of the transported fluid. This development will be proposed for future investigations for road tankers of circular section and other types of section.

\section{REFERENCES}

[1] Rajagounder, R., Mohanasundaram, G. V., \& Kalakkath, P. (2016). A study of liquid sloshing in an automotive fuel tank under uniform acceleration. Engineering Journal, 20(1), 7185. https://doi.org/10.4186/ej.2016.20.1.71

[2] Gillespie, T. D. (1992). Fundamentals of Vehicle Dynamics. 7th. ed. Warrendale, PA.: SAE International, 1992. ISBN 1560911999. https://doi.org/10.4271/R-114

[3] Rill, G. (2011). Road vehicle dynamics: fundamentals and modeling. CRC Press. https://doi.org/10.1201/9781439897447

[4] Moreno, G., Vieira, R., \& Martins, D. (2018). Highway designs: effects of heavy vehicles stability. Dyna, 85(205), 205-210. https://doi.org/10.15446/dyna.v85n205.69676

[5] Ranganathan, R. (1993). Rollover threshold of partially filled tank vehicles with arbitrary tank geometry. Proceedings of the Institution of Mechanical Engineers, Part D: Journal of Automobile Engineering, 207(3), 241-244. https://doi.org/10.1243/PIME_PROC_1993_207_185_02

[6] Winkler, C. (1999). Rollover of Heavy Commercial Vehicles. UMTRI Research Review. The University of Michigan Transportation Research Institute, 31(4), 120.

[7] Prem, H., Mai, L., \& Brusza, L. (2006). Tilt testing of two heavy vehicles and related performance issues. In International Symposium on Heavy Vehicle Weights and Dimensions, 9th.

[8] Moreno, G., Nicolazzi, L. C., Vieira, R. D. S., \& Martins, D. (2017). Suspension and tyres: stability of heavy vehicles. 
International Journal of Heavy Vehicle Systems, 24(4), $305-$ 326. https://doi.org/10.1504//JHVS.2017.087221

[9] Cengel Yunus, A. \& Cimbala John, M. (2006). Fluid Mechanics Fundamentals and Applications. Editorial McGraw Hill.

[10] Dasgupta, A. (2011). Effect of tank cross-section and longitudinal baffles on transient liquid slosh in partly-filled road tankers. Doctoral dissertation, Concordia University.

[11] Davies, T. H. (1983). Mechanical networks-III wrenches on circuit screws. Mechanism and Machine Theory, 18(2), 107112. https://doi.org/10.1016/0094-114X(83)90102-7

[12] Davies, T. H. (2000). The 1887 committee meets again. Subject: freedom andconstraint. Ball 2000 Conference, Cambridge University Press, Trinity College, Cambridge, UK., p. 56.

[13] Tsai, L. W. (1999). Robot Analysis-The Mechanism of Serial and Paralle Manipulators. New York: John Wiley \& Sons. ISBN 0-471-32593-7.

[14] ISO-14792. (2011). Heavy commercial vehicles and buses Steady state circular tests. International Organization for Standardization. Geneva, Switzerland.

[15] Woodrooffe, J., Sweatman, P., Arbor, A., Middleton, D., James, R., \& Billing, J. R. (2010). National Cooperative Highway Research Program NCHRP. Report 671. Review of Canadian Experience with the Regulation of Large Commercial Motor Vehicles. Ed. National Academy of Sciences, Washington, D.C., ISBN 978-0-309-15518-2. https://doi.org/10.17226/14458

[16] Walker, H. K. \& Pearson, J. R. (1987). Recommended regulatory principles for interprovincial heavy vehicle weights and dimensions. Tech. rep., CCMTA/RTAC Vehicle Weights and Dimensions Study Implementation Committee Report.

Contact information:

Gonzalo Guillermo MORENO CONTRERAS, PhD

(Corresponding author)

University of Pamplona, Pamplona, Colombia

Department of Mechanical, Mechatronic and Industrial Engineering

Faculty of Engineering, University of Pamplona, Pamplona, Colombia

Postal address: 543050

E-mail: gmoren@unipamplona.edu.co

Juan Carlos SERRANO RICO, PhD

University of Pamplona, Pamplona, Colombia

Department of Mechanical, Mechatronic and Industrial Engineering,

Faculty of Engineering, University of Pamplona, Pamplona, Colombia

Postal address: 543050

E-mail: jcserrano@unipamplona.edu.co

Bladimir RAMON VALENCIA, PhD

University of Pamplona, Pamplona, Colombia

Department of Mechanical, Mechatronic and Industrial Engineering

Faculty of Engineering, University of Pamplona, Pamplona, Colombia

Postal address: 543050

E-mail: hbladimir@unipamplona.edu.co 